\title{
Relationship between posterior pharyngeal pouch and hiatus hernia
}

\author{
T. B. SMILEY, P. K. CAVES ${ }^{1}$, and D. C. PORTER \\ Royal Victoria Hospital, Belfast, Northern Ireland
}

\begin{abstract}
The formation of posterior pharyngeal pouches is generally attributed to dysfunction of the cricopharyngeal sphincter. The reason for this dysfunction and its exact nature have not been established. Observations in the Royal Victoria Hospital, Belfast, suggested that an association exists between pharyngeal pouch and hiatus hernia. Barium studies performed in patients with a pharyngeal pouch using a described technique demonstrated the presence of a hiatus hernia in 32 out of 34 patients.

Illustrative case histories and a review of published work on cricopharyngeal function are provided to support our conclusions that gastro-oesophageal reflux is the primary factor leading to cricopharyngeal dysfunction, with the formation of a pharyngeal pouch in some cases. Several possible criticisms of this theory are discussed and the need for further investigation in patients with a pharyngeal pouch is emphasized. The management of patients with a pharyngeal pouch and hiatus hernia is briefly outlined.
\end{abstract}

Diverticulum of the posterior pharyngeal wall was first described by Ludlow in 1769. Zenker's name became associated with this condition after he and von Ziemssen had described it more completely in 1874. Since then much has been written about 'pharyngeal pouches' and, in particular, their aetiology. The formation of posterior pharyngeal pouches is generally attributed to dysfunction of the cricopharyngeal sphincter (Negus, 1957). It is postulated that a bolus of food, passing through the lower pharynx during the second stage of swallowing, is held up partially or completely at the sphincter which has failed to open in a co-ordinated manner. The pressure in the lower pharynx rises, and the mucosa is herniated through the posterior wall, where a weak point between the oblique and horizontal fibres of the inferior constricter is said to exist (Killian's dehiscence).

Some authors have attributed the dysfunction to achalasia of the sphincter, a concept based mainly on their operative findings (Sutherland, 1962; Thompson, 1965; Belsey, 1966). Negus (1957) suggested that chronic inflammatory changes, in the region of the sphincter, might prevent it from opening properly. On the basis of cineradiographic studies, Ardran and Kemp (1961) and Lund (1968) maintained that the 'Requests for reprints to. P.K.C., Brompton Hospital, London,
S.W.3 sphincter relaxed normally in patients with a pouch, but closed too soon before the last part of the bolus had passed through. Wilson (1962) stated that patients with a pharyngeal pouch had an abnormally large pharynx and he believes that when a single, normal swallow does not completely empty the pharynx, the patient develops a 'secondary swallow'. This finds the cricopharyngeus contracted, and the rise in pressure in the lower pharynx results in the formation of a pulsion diverticulum.

We have subscribed to the view that some form of incoordination or 'spasm' of the cricopharyngeus is present in patients who develop a pharyngeal pouch but have never found any satisfactory reason given in the literature to explain its occurrence. It was then noticed that some patients with a pharyngeal pouch had evidence of a hiatus hernia on their barium studies. Further investigation by Hunt, Connell, and Smiley (1970) in this hospital showed that the resting pressure of the cricopharyngeal sphincter was high in patients with gastro-oesophageal reflux, and in most patients with a pharyngeal pouch. A careful radiological examination of the cardia region was therefore performed on every patient with a pharyngeal pouch to determine if hiatus hernia and gastrooesophageal reflux were present. The results of this survey are reported, with our reasons for believing that gastro-oesophageal reflux is of pri- 
mary importance in the development of a pharyngeal pouch.

\section{MATERIAL AND METHODS}

Thirty-four patients who had pharyngeal pouches were examined. Nearly all of them had surgical treatment in the Thoracic Surgical Unit, Royal Victoria Hospital, Belfast. The average age of the patients was 63 (range 44 to 84 ) years. There were 23 males and 11 females.

Most of the radiological examinations were carried out by one of us (D.C.P.) using exactly the same technique in each case.

Barium emulsion, $175 \mathrm{ml}$., is taken by the patient in the erect position. The table is then tilted to the horizontal plane so that the patient is supine. Reflux in this posture is unusual. The patient is then turned on to the right side and at a varying angle-sometimes only a few degrees and seldom more than $45^{\circ}$-reflux and the hernia, if present, will often be seen. If at this stage reflux and/or herniation have not been demonstrated, the patient is asked to swallow saliva. This determined swallowing effort results in oesophageal peristalsis which, on reaching the cardia, produces relaxation of the physiological sphincter. If the factors necessary for herniation are present, such as a wide lax hiatus and increased intra-abdominal pressure, then herniation and reflux should occur. To demonstrate the reflux and the herniated loculus on film it is often an advantage to give the patient barium cream to swallow at this stage, and to rotate the patient even further to the right until almost prone.

Placing the patient in the Trendelenburg position is of little value and abnormal posturing over bolsters -postures never assumed by the average patient- have not been found helpful or desirable. Stooping, by increasing intra-abdominal pressure, has occasionally demonstrated a hernia and reflux, when the right supine oblique posture has failed. Herniation may become apparent as the stooping patient resumes the erect posture. This may be due to the extra intraabdominal pressure or to closure of the glottis at the end of a deep breath-which has been shown to result in relaxation of the hiatus.

\section{RESULTS}

A hiatus hernia was found in all but two of the patients examined (Table).

\section{T A B L E}

INCIDENCE OF HIATUS HERNIA IN PATIENTS WITH A PHARYNGEAL POUCH

\begin{tabular}{|c|c|c|c|}
\hline $\begin{array}{l}\text { Pharyngeal pouch and hiatus hernia } \\
\text { Pharyngeal pouch-no hernia demonstrated }\end{array}$ & $\ddot{*}$ & 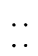 & $\begin{array}{r}32 \\
2\end{array}$ \\
\hline Total number of patients .. & .. & . & 34 \\
\hline
\end{tabular}

In most patients the hernia and reflux were demonstrated fairly easily (Figs 1 and 2) but only rarely could both the pouch and hernia be shown on the same film (Fig. 3). No connection existed between the size of the pouch and the degree of refiux demonstrated (Figs 4 and 5).

The importance of the hiatus hernia in the development and management of the pharyngeal pouch will be seen from the following illustrative case histories:

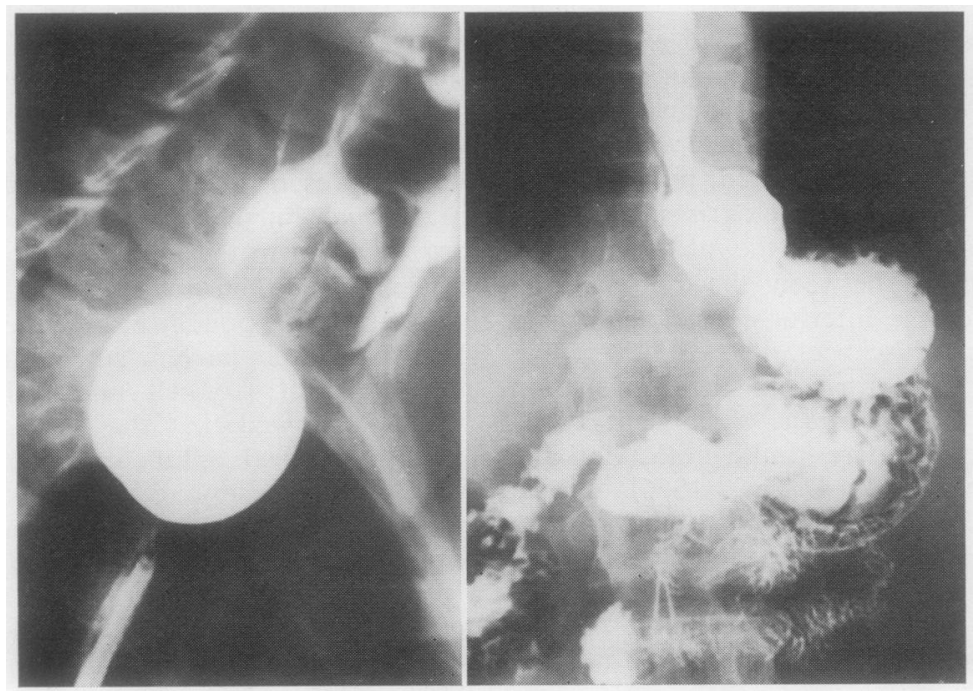

FIG. 1. A-P view of pharyngeal pouch and hiatus hernia in same patient. 


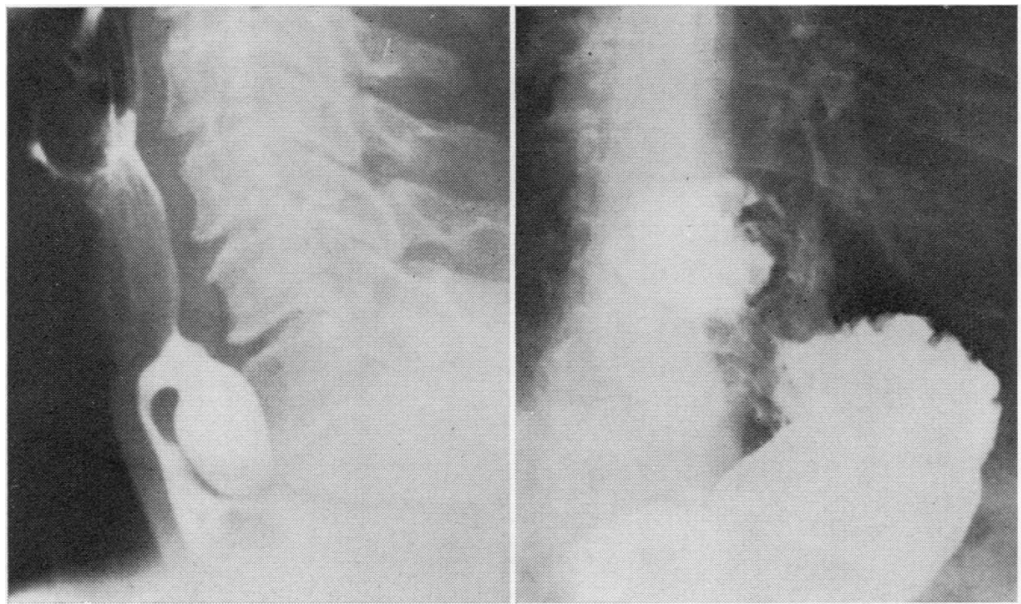

FIG. 2. Lateral view of pharyngeal pouch and hiatus hernia in same patient.

CASE 1 M.G. aged 51 years, while recovering from a myocardial infarction, noticed difficulty in swallowing. This became more severe, and within a few months he had a bad taste in the mouth and regurgitated food sometimes recognized as having been eaten a few days earlier, e.g., tomato skin. Barium swallow showed a pharyngeal pouch and a hiatus hernia with gastro-oesophageal reflux.

The pouch was excised without division of the cricopharyngeus muscle. The patient's dysphagia was improved and barium swallow was satisfactory. Eighteen months later dysphagia returned and a barium swallow showed a recurrence of the pouch. His car-

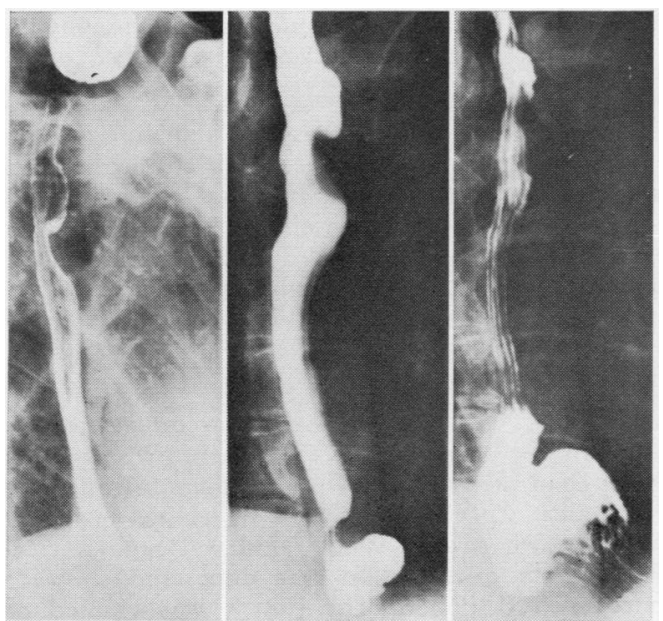

FIG. 3. Case 2. Pharyngeal pouch and hiatus hernia shown on same film. diac condition was now satisfactory. The pouch was again excised, the cricopharyngeus muscle divided and the hiatus hernia repaired.

CASE 2 Mrs. McB. aged 60 years complained of heartburn and cervical dysphagia. The heartburn had been present for some years and the dysphagia for a few months. Barium swallow showed a hiatus hernia with reflux and a pharyngeal pouch, which the radiologist was able to demonstrate on a single film (Fig. 3).

The patient was very keen to have not only the dysphagia but also the heartburn treated. Under the

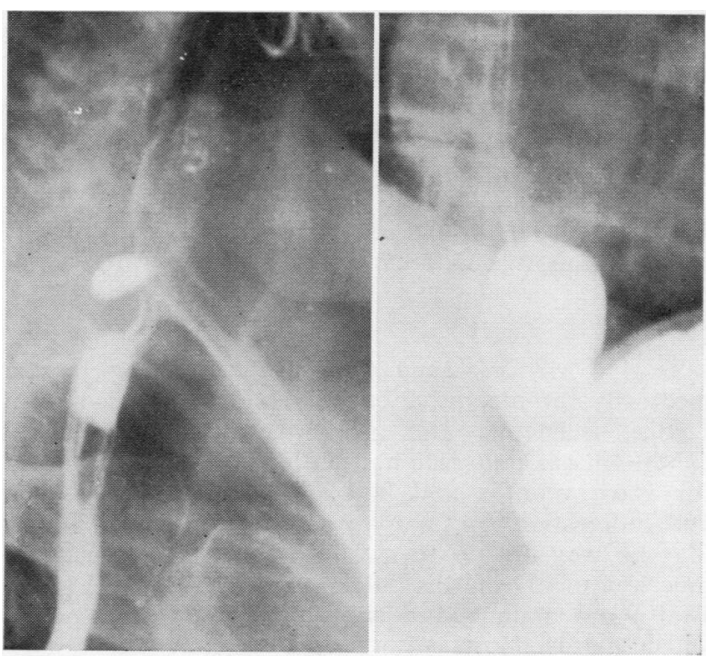

FIG. 4. Case 3. Small pharyngeal pouch and large hiatus hernia in same patient. 


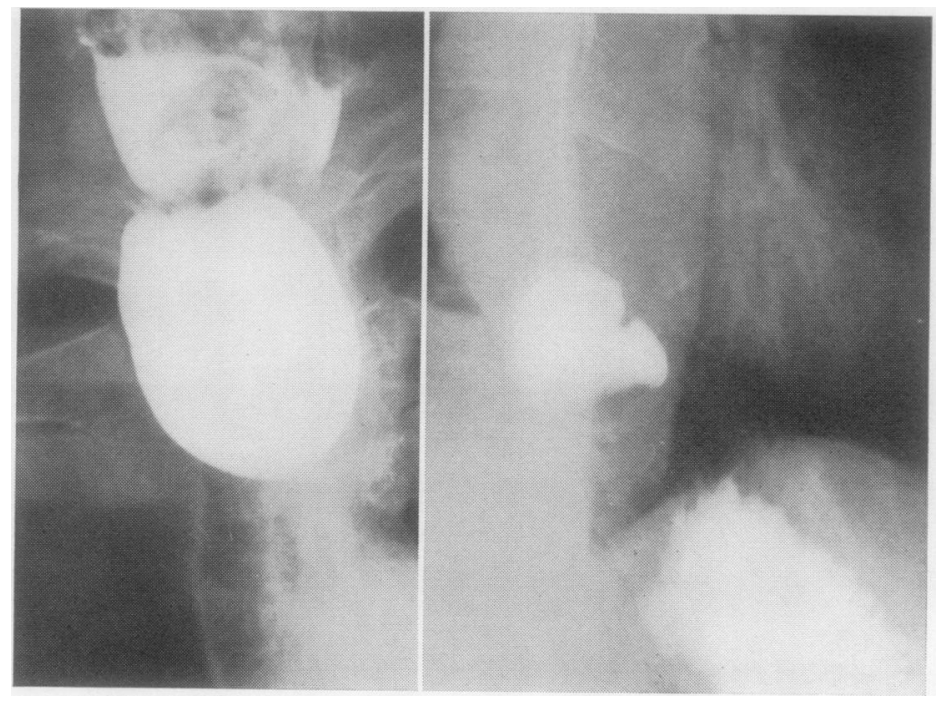

FIG. 5. Large pharyngeal pouch and hiatus hernia in same patient.

same general anaesthetic, the pouch was excised and the cricopharyngeal muscle divided through a transverse incision in the neck; the hiatus hernia was then repaired through a left thoracotomy.

CASE 3 Mrs. D. aged 54 years had a severe 'choking attack'. During the next month she had severe cervical dysphagia. She admitted to indigestion for some years, but it had never been severe enough for her to seek medical advice. Barium swallow showed a small pharyngeal pouch and a moderate-sized hiatus hernia with gastro-oesophageal reflux (Fig. 4). Cricopharyngeal pressure studies were done and found to be abnormally high $\left(62 \mathrm{~cm} . \mathrm{H}_{2} \mathrm{O}\right)$. The hiatus hernia only was repaired. Dysphagia rapidly improved and the patient was eating a normal diet before leaving hospital on the twelfth post-operative day. Six months later the cricopharyngeal pressure was $27 \mathrm{~cm} . \mathrm{H}_{2} \mathrm{O}$. Two years later the patient remains well. She still has a small pharyngeal pouch which is not causing symptoms.

CASE 4 Mr. W. aged 63 years was being treated medically for ulcerative colitis. He also suffered from cardiac ischaemia and urinary calculi. His general condition was poor. He had noticed cervical dysphagia for some months and had suffered from heartburn and indigestion for many years. When it was found that he was unable to swallow large tablets used in the treatment of his ulcerative colitis, a barium swallow was done and showed a pharyngeal pouch and a hiatus hernia with reflux.

To facilitate the medical treatment it was decided to excise the pouch. This was done and the crico- pharyngeus muscle was divided. On the sixth postoperative day he was swallowing well, apart from slight pain. On the ninth post-operative day his neck suddenly became full in the region of the wound, and food was found to be present when the wound was drained. The presence of an oesophago-cervical fistula was confirmed at oesophagoscopy, which also demonstrated gross gastro-oesophageal reflux. The patient was tube fed and propped up in bed. The ulcerative colitis and his general condition deteriorated. He was unable to remain propped up for long periods, and while lying prone, it was found that he had a bile-stained discharge from the sinus. Six weeks later he died from bronchopneumonia, possibly due to aspiration of oesophageal contents.

CASE 5 Dr. M. aged 82 years had had the symptoms of a hiatus hernia for many years. It had been demonstrated by barium swallow 10 years previously. No pharyngeal pouch was present at that examination. For one year he had cervical dysphagia and a barium swallow showed a pouch and hiatus hernia. The pouch was excised and the cricopharyngeal muscle divided, with relief of the dysphagia.

CASE 6 Dr. L. had suffered from indigestion and heartburn for some years. Two barium meal examinations had been done without demonstrating any abnormality. Cervical dysphagia developed insidiously but was never severe. When food was regurgitated a third barium examination was done and showed a moderate-sized pouch. The pouch was excised and the cricopharyngeus was divided with relief of the dysphagia. He continues to suffer from heartburn. 


\section{DISCUSSION}

It is well established that the passage of a bolus of food through the pharynx is due to a peristaltic wave of contraction passing down the pharyngeal muscles (Fyke and Code, 1955). Simultaneously the larynx and pharynx are elevated by the vertical muscles so as to 'engulf' the bolus (Negus, 1957; Localio and Stahl, 1968). With the arrival of the bolus in the lower pharynx the cricopharyngeal sphincter relaxes reflexly to permit its passage through into the upper oesophagus. Negus believed that the afferent arc of this reflex began in sensory fibres from the lower pharynx. He thought that failure of the cricopharyngeus to relax and the formation of a pouch could result from loss of sensation in this region. On the basis of cineradiographic studies, Dohlman and Mattsson (1959) believed the afferent arc to commence in stretch receptors in the cricopharyngeus which were stimulated by the elevation of the larynx during swallowing. They suggested that in elderly people the posterior pharyngeal wall becomes detached from the pre-vertebral fascia. When the larynx rises during swallowing the cricopharyngeus rises with it, the stretch receptors are not stimulated and so the sphincter does not relax. Thus there is not only a build up of pressure in the lower pharynx but a potential space at negative pressure is created behind the pharynx into which the mucosa is both sucked and forced. Others have suggested that paralytic neural defects interfere with the reflex arc, and prevent relaxation of the sphincter. Lewis and Edwards (1962) point out that the cricopharyngeus has a double motor supply from vagal fibres arising in both the nucleus ambiguus and the dorsal nucleus of the vagus and is thus very rarely affected even in bulbar palsy. Paralysis of the cricopharyngeus has never been demonstrated in patients with a pharyngeal pouch.

Using a tiny pressure transducer, Fyke and Code (1955) showed that the resting pressure in the cricopharyngeal sphincter was normally 20 to $30 \mathrm{~cm}$. of water higher than the pressure in the upper oesophagus or lower pharynx. In the period of reflex relaxation during swallowing the sphincter pressure fell to an average of $-12 \mathrm{~cm}$. of water, then rose to a mean maximal pressure of $92 \mathrm{~cm}$. of water before returning to normal. Atkinson, Kramer, Wyman, and Ingelfinger (1957), using fluid-filled open-tipped tubes, found a mean resting pressure in the sphincter of $35 \mathrm{~cm}$. of water. This pressure dropped to equal that in the upper oesophagus on swallowing, then rose again to its resting level or even higher.
The first study on cricopharyngeal function in patients with a posterior pharyngeal pouch using pressure manometry was by Kodicek and Creamer (1961). Using fluid-filled open-tipped tubes they studied five patients with a pharyngeal pouch and found that the pharynx and the cricopharyngeal sphincter appeared to behave in a normal fashion. They found the resting sphincteric pressure to be normal for elderly patients with a mean value of $20 \mathrm{~cm}$. of water. Doubt has been expressed as to the validity of their findings (Lewis and Edwards, 1962) and completely contrary results were found by Hunt et al. (1970), who found a mean resting pressure of $14.6 \mathrm{~cm}$. of water in normal subjects and a mean resting pressure of $41.0 \mathrm{~cm}$. of water in five patients with a pharyngeal pouch (Fig. 6). Significantly, in patients with gastro-oesophageal reflux, the mean resting pressure was $32 \cdot 1 \mathrm{~cm}$. of water and in patients with reflux who complained of cervical dysphagia the mean resting cricopharyngeal pressure was $37.9 \mathrm{~cm}$. of water. Following repair of hiatus hernia the mean resting pressure fell to $21.4 \mathrm{~cm}$. of water. The resting cricopharyngeal pressure was highest in the patient with the smallest pouch (Fig. 4) and was almost normal in one patient with a huge pouch (Fig. 7).

These findings led to the radiological survey already described. The finding of 32 hernias in 34 patients with pharyngeal pouches is very significant. Some patients were known to have had a hiatus hernia for years before they presented with dysphagia and, on investigation, a pouch was demonstrated (e.g., case 5). In others (e.g., cases 2 and 4) gastro-oesophageal reflux symptoms were presenting features. In the second case both the hernia and pouch were dealt with at the same time but, in case 4 , the hernia was not operated on because of his poor general health. Unfortunately, the wound broke down and bilestained gastric contents welled out of the cervical fistula until his death. The first case was known to have a hernia with symptoms at the time of the excision of the pouch. As little as possible was done in view of his myocardial state and the cricopharyngeus was not divided. As a result the pouch recurred and required re-excision at the time of the hiatus hernia repair...Most conclusive of all is case 3, whose pouch has not been touched but whose dysphagia completely disappeared when the hiatus hernia was repaired. Of the two patients in whom hernias could not be demonstrated, one (case 6) has had severe symptoms of gastro-oesophageal reflux for years.

From these findings we have concluded that 


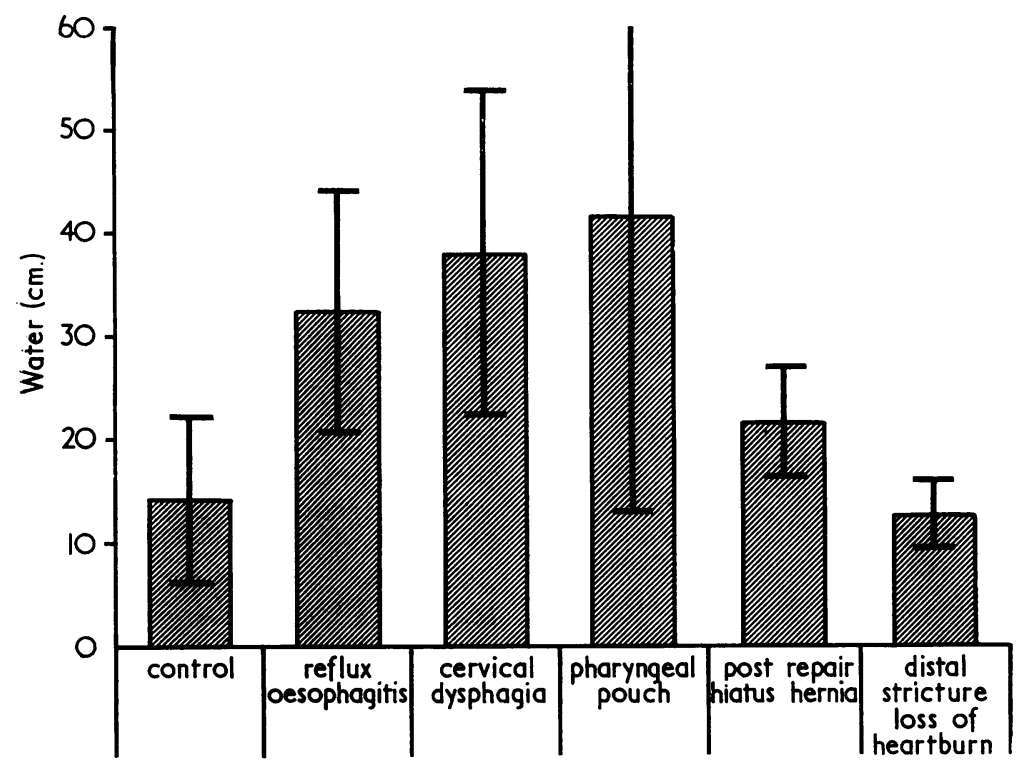

FIG. 6. Mean and standard deviation of the resting cricopharyngeal pressure in patients with oesophageal disorders compared with a control group. (Reprinted from Gut, 11, 303, 1970, by permission of the author, the Editor and the Publisher, B.M.A. House, Tavistock Square, London, W.C.1.)

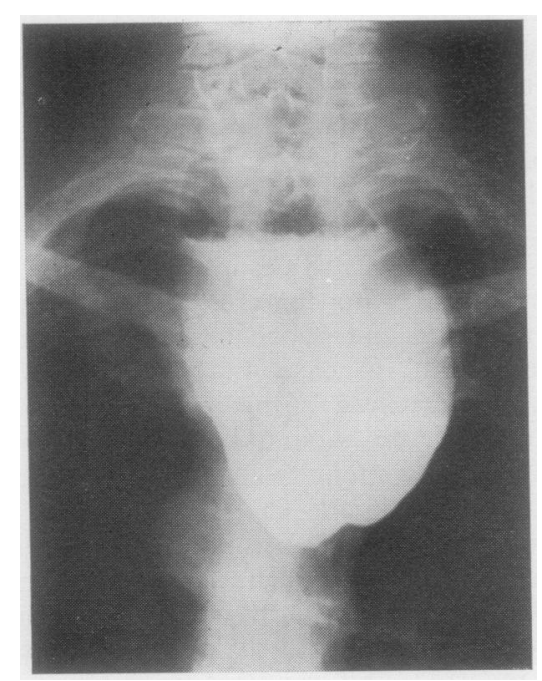

FIG. 7. Huge pharyngeal pouch containing a whole measure of barium emulsion.

gastro-oesophageal reflux is the cause of the cricopharyngeal dysfunction which is the initiating factor in the formation of a pharyngeal pouch.

There remain several difficulties with this theory. Hiatus hernia with reflux is a common condition while posterior pharyngeal pouch is relatively rare. Obviously not all patients with gastro-oesophageal reflux develop such cricopharyngeal dysfunction that a pouch results. Pharyngeal pouch is particularly rare in patients under 40 years of age and, as the incidence of hiatus hernia also increases with age, it may be that insufficient time is available after the development of reflux for a pouch to be produced.

Hiatus hernia is also commoner in women but pharyngeal pouch is commoner in men, the usual sex incidence being male 2-3: female 1 . King (1947) suggested that the male pharynx is bigger and more muscular than the female and it may be that, in the presence of an obstruction, a man makes more vigorous swallowing efforts than a woman and produces a higher pressure in the lower pharynx.

A more important criticism is that the pressure in the lower pharynx on swallowing has not been investigated in patients with a pharyngeal pouch. A raised resting cricopharyngeal pressure, on its own, cannot be the cause of pharyngeal pouch formation. We believe that the higher, resting cricopharyngeal pressure in patients with a pouch does not prevent the sphincter from relaxing in a normal fashion but that the sphincter closes too soon before the pressure in the lower pharynx 
has returned to normal. We hope to be able to clarify this point after further studies.

It has been suggested that perhaps it is the pharyngeal pouch which produces the hiatus hernia. We can think of no logical reasoning as to how this might come about and, in our clinical experience, the hiatus hernia and/or its symptoms have been present before the pouch in almost every case, usually for many years.

Some authors have believed pharyngeal pouches to be congenital in origin. The faci that they are usually seen in old age must indicate an acquired origin (Clagett, 1963; Payne and Clagett, 1965) and our theory would support this.

It remains to be explained why this relationship between hiatus hernia and pharyngeal pouch has not previously been recognized. Parrish (1968) reported four patients with dysphagia due to cricopharyngeal dysfunction and noted that one had a hiatus hernia with gastro-oesophageal reflux. In reporting 37 cases of pharyngeal pouch, Kinley (1966) noted that two had also a hiatus hernia. Belsey (1966) recognized the occurrence of spasm of the cricopharyngeus in patients with gastrooesophageal reflux and discussed the theoretical danger of dividing the cricopharyngeal sphincter when excising a pharyngeal pouch should reflux be present. No previous attempt to examine the cardiac region appears to have been made during barium studies on patients with a pharyngeal pouch. The obvious difficulty is that a whole measure of barium emulsion may be retained in a large pouch (Fig. 7) and, having found such a pouch, the lower end of the oesophagus may have been regarded as of secondary interest. Some patients with a pouch find it impossible to swallow sufficient contrast to enable the cardia and fundus to be outlined properly and, in these cases, radiological examination of the cardia must be deferred until after excision of the pharyngeal pouch and division of the cricopharyngeus.

We regard one-stage excision of the pouch with division of the cricopharyngeus as the treatment of choice for a pharyngeal pouch.

Our only recurrence (case 1) was after excision of the pouch alone, accomplished without damage to the cricopharyngeus. It has become our practice to repair the hiatus hernia in those patients who have demonstrable reflux and who are fit enough for this operation. In patients (e.g., case 3) in whom the pouch is small, it may be sufficient only to repair the hiatus hernia.

Our thanks are due to $\mathrm{Mr}$. J. Bingham and $\mathrm{Mr}$. H. M. Stevenson for permission to include some of their cases in this report; and to Miss N. Sewell for typing the manuscript.

\section{REFERENCES}

Ardran, G. M., and Kemp, F. H. (1961). The radiography of the lower lateral food channels. J. Laryng., 75, 358.

Atkinson, M. Kramer, P., Wyman, S. M., and Ingelfinger, F. J. (1957). The dynamics of swallowing. I. Normal pharyngeal mechanisms. J. clin. Invest., 36, 581.

Belsey, R. (1966). Functional disease of the oesophagus. J. thorac. cardiovasc. Surg., 52, 164.

Clagett, O. T. (1963). Pharyngo-esophageal diverticula. Surg. Clin. N. Amer., 43, 1165.

Doblman, G., and Mattsson, O. (1959). The role of the cricopharyngeal muscle in cases of hypopharyngeal diverticula. Amer. J. Roentgenol., 81, 561 .

Fyke, F. E., and Code, C. F. (1955). Resting and deglutition pressures in the pharyngo-esophageal region. Gastroenterology, 29, 24.

Hunt, P. S., Connell, A. M., and Smiley, T. B. (1970). The cricopharyngeal sphincter in gastric reflux. Gut, 11, 303.

King, B. T. (1947). New concepts of the etiology and treatment of diverticula of the esophagus. Surg. Gynec. Obstet., 85, 93.

Kinley, C. E. (1966). The management of hypopharyngeal diverticulum. Canad. J. Surg., 9, 27.

Kodicek, J., and Creamer, B. (1961). A study of pharyngeal pouches. J. Laryng., 75, 406.

Lewis, R. S., and Edwards, W. G. (1962). The treatment of pharyngeal diverticula. Brit. J. Surg., 50, 1.

Localio, S. A., and Stahl, W. M. (1968). Diverticular disease of the alimentary tract. Part II: The esophagus, stomach, duodenum and small intestine. Curr. Probl. Surg., January, pp. 5-13.

Ludlow, A. (1769). A case of obstructed deglutition, from a preternatural dilation of, and bag formed in the pharynx. Med. Observations and Inquiries, by a Society of Physicians in London, $3,85$.

Lund, W. S. (1968). The cricopharyngeal sphincter: its relationship to the relief of pharyngeal paralysis and the surgical treatment of the early pharyngeal pouch. J. Laryng., 82, 353 .

Negus, V. E. (1957). The etiology of pharyngeal diverticula. Bull. Johns Hopk. Hosp., 101, 209.

Parrish, R. M. (1968). Cricopharyngeus dysfunction and acute dysphagia. Canad. med. Ass. J., 99, 1167.

Payne, W. S., and Clagett, O. T. (1965). Pharyngeal and esophageal diverticula. Curr. Prob. Surg., April, pp. 4-16.

Sutherland, H. D. (1962). Cricopharyngeal achalasia. J. thorac. cardiovasc. Surg., 43, 114.

Thompson, H. T. (1965). Cricopharyngeal achalasia. N.Z. med. J., 64, 22.

Wilson, C. P. (1962). Pharyngeal diverticula, their cause and treatment. J. Laryng., 76, 151.

Zenker, T. A., and Ziemssen, H. von (1874). Krankheiten des Oesophagus. Handbuch der speziellen Pathologie und Therapie, Vol. 7. pp. 50-87. Vogel, Leipzig. 